\title{
Cateter central de inserção periférica em neonatologia: Revisão integrativa
}

\author{
Central peripheral insertion catheter in neonatology: Integrative review \\ Catéter de inserción periférica central en neonatología: Revisión integrativa
}

Recebido: 04/07/2021 | Revisado: 13/07/2021 | Aceito: 15/07/2021 | Publicado: 15/07/2021

Andreize de Freitas Ramos
ORCID: https://orcid.org/0000-0002-0765-2787
Santa Casa de Misericórdia de Porto Alegre, Brasil
E-mail: afr.enf@rede.ulbra.br
Sheila Beatris Kochhann
ORCID: https://orcid.org/0000-0002-0841-0931
Santa Casa de Misericórdia de Porto Alegre, Brasil
E-mail: Sheila_kochhann@ hotmail.com
Jonas Hantt Corrêa Lima
ORCID: https://orcid.org/0000-0001-5672-1024
Universidade Luterana do Brasil, Brasil
E-mail: jonashantt@hotmail.com
Patricia Mara Guralski Secco
ORCID: https://orcid.org/0000-0002-2611-7800
Universidade Luterana do Brasil, Brasil
E-mail: patriciamaragsecco@gmail.com
Fabrício Wilsmann Curi Pereira
ORCID: https://orcid.org/0000-0002-9479-7529
Universidade Luterana do Brasil, Brasil
E-mail: fabricio.w.curi@gmail.com
Carolina Scheer Ely
ORCID: https://orcid.org/0000-0002-8338-2213
Universidade Luterana do Brasil, Brasil
E-mail: talesbw@hotmail.com
ORCID: https://orcid.org/0000-0003-4885-7866
Universidade Luterana do Brasil, Brasil
E-mail: carolinaely@rede.ulbra.br
Bruna Reis Krug
E-mail

\section{Resumo}

O Cateter central de inserção periférica passou a ser utilizado no Brasil na década de 1990 e tem sido amplamente utilizado em neonatologia e pediatria, sendo o mesmo cada vez escolhido como dispositivo de terapia venosa de longa duração em neonatologia. Esse procedimento deve ser realizado por profissionais treinados e capacitados por ser uma técnica mais sensível em neonatos. O presente estudo foi desenvolvido através da uma revisão integrativa, tendo como objetivo geral conhecer o que a literatura discorre acerca do uso de cateter central de inserção periférica em neonatologia. Após análise minuciosa dos artigos estudados eles foram segregados em categorias estabelecidas levando em consideração seus objetivos, sendo que as categorias geradas foram: Complicações causadas pelo uso do cateter central de inserção periférica (CCIP) e custo com o uso do cateter. O mal posicionamento foi a complicação mais relatada nos artigos estudados seguido por dor e infecções de corrente sanguínea. O elevado custo do procedimento é um assunto muito evidenciado nos artigos estudados, todavia não foram encontrados artigos que calculassem esse custo no Brasil, todos utilizavam valores internacionais. Não foram localizados artigos que contemplassem a indicação e contraindicação para o uso do CCIP. 
Palavras-chave: Neonatologia; Cateter central de inserção periférica; Enfermagem.

\begin{abstract}
The Peripherally Inserted Central Catheter came to be used in Brazil in the 1990s and has been widely used in neonatology and pediatrics, being increasingly chosen as a device for long-term venous therapy in neonatology. This procedure must be performed by trained and qualified professionals as it is a more sensitive technique in neonates. The present study was developed through an integrative review, with the general objective of knowing what the literature discusses about the use of peripherally inserted central catheters in neonatology. After a thorough analysis of the studied articles, they were segregated into established categories considering their objectives, and the categories generated were Complications caused using peripherally inserted central catheter (PICC) and cost of using the catheter. Mispositioning was the most reported complication in the articles studied, followed by pain and bloodstream infections. The high cost of the procedure is a subject very evident in the articles studied, however, no articles were found that calculated this cost in Brazil, all of which used international values. No articles were found that contemplated the indication and contraindication for the use of the PICC.
\end{abstract}

Keywords: Neonatology; Peripherally inserted central catheter; Nursing.

\title{
Resumen
}

El catéter central de inserción periférica se empezó a utilizar en Brasil en la década de 1990 y se ha utilizado ampliamente en neonatología y pediatría, siendo cada vez más elegido como dispositivo para la terapia venosa a largo plazo en neonatología. Este procedimiento debe ser realizado por profesionales capacitados y calificados ya que es una técnica más sensible en neonatos. El presente estudio se desarrolló mediante una revisión integradora, con el objetivo general de conocer lo que discute la literatura sobre el uso de catéteres centrales de inserción periférica en neonatología. Luego de un análisis exhaustivo de los artículos estudiados, se segregaron en categorías establecidas teniendo en cuenta sus objetivos, y las categorías generadas fueron: Complicaciones por el uso de catéter central de inserción periférica (CCIP) y costo de uso del catéter. El mal posicionamiento fue la complicación más reportada en los artículos estudiados, seguida del dolor y las infecciones del torrente sanguíneo. El alto costo del procedimiento es un tema muy evidente en los artículos estudiados, sin embargo, no se encontraron artículos que calculen este costo en Brasil, todos los cuales utilizaron valores internacionales. No se encontraron artículos que contemplen la indicación y contraindicación para el uso del PICC.

Palabras clave: Neonatología; Catéter central de inserción periférica; Enfermería.

\section{Introdução}

O Cateter Central Inserido Perifericamente (CCIP) foi descrito pela primeira vez na literatura científica, em 1929, como forma de acessar de forma periférica o sistema venoso central do paciente. O médico Forssmann realizou o procedimento se autocateterizando com uma sonda uretral, através de uma veia em sua fossa cubital. Em meados de 1970 foi então desenvolvido cateteres de silicone que foram de forma inicialmente utilizados em centros de terapia intensiva neonatal, todavia a expansão do uso desse cateter foi somente na década de 80 (Philpot \& Griffiths, 2003).

O CCIP passou a ser utilizado no Brasil na década de 1990 e tem sido amplamente utilizado em neonatologia e pediatria, sendo o mesmo preferencialmente escolhido como dispositivo de terapia venosa de longa duração em neonatologia, esse procedimento deve ser realizado por profissionais treinados e capacitados por ser uma técnica ainda mais sensível em neonatos (Toma \& Tsunechiro, 2003).

Os recém-nascidos (RNs) que são submetidos a inserção de CCIP são em suma, prematuros de baixo peso ao nascer que necessitam de uma forma mais que adequada para garantir o seu desenvolvimento (Fricke et al., 2005). Segundo o Conselho Federal de Enfermagem, o procedimento é vedado a enfermeiros e médicos, porém eles devem participar de treinamentos que incluam conteúdos teóricos e práticos. Os conteúdos da capacitação devem incluir: inserção, manutenção, retirada além de indicações e contraindicações (Cofen, 2017).

\section{Metodologia}

Este artigo tem como objetivo principal apresentar um assunto que vem exponencialmente sendo difundido no âmbito científico e hospitalar através das literaturas existentes nas bases de dados científicas. O aumento progressivo vem sendo 
justificado pelo envolvimento da enfermagem em procedimentos de maior complexidade e, consequentemente, a especialização do profissional graduado em enfermagem que tem crescido cada vez mais.

Assim, o presente estudo foi desenvolvido através da uma revisão integrativa, tendo como objetivo geral conhecer o que a literatura discorre acerca do uso de cateter central de inserção periférica em neonatologia.

A revisão integrativa (RI), é um dos métodos de pesquisa utilizados na Pesquisa Baseada em Evidências (PBE), inclui a análise de pesquisas relevantes que dão suporte para a tomada de decisão e, a melhoria da prática clínica, possibilitando a síntese do estado do conhecimento de um determinado assunto, além de apontar lacunas do conhecimento que precisam ser preenchidas com a realização de novos estudos (Souza, Silva \& Carvalho, 2010).

Essa metodologia é realizada em seis etapas: identificação do tema; definição dos critérios de inclusão e exclusão dos artigos e escolha das bases de dados; identificação dos estudos selecionados; síntese dos estudos; análise e interpretação dos dados coletados e, apresentação dos resultados (Minayo, 2013).

A questão norteadora dessa pesquisa foi buscar publicações para analisar e sintetizar o conhecimento acerca do cateter central de inserção periférica em neonatologia.

Os critérios de inclusão estabelecidos foram: artigos científicos publicados, no ano de 2016 a 2021. Considerou-se como critérios de Inclusão os temas relacionados a inserção do CCIP em pacientes neonatais, sendo excluídos os que antecedem o ano de 2016 e os que abordavam assuntos relacionados a cateter venoso central que não fosse realizado por inserção periférica assim como demais assuntos que não se encaixavam nos critérios de inclusão.

O período de coleta de dados ocorreu de 25 de dezembro de 2020 a 09 de janeiro de 2021, utilizando descritores, obtidos nos Descritores em Ciências da Saúde (DeCS) "Neonatologia" ("neonatology"). Também foi utilizado um descritor não controlado: "Cateter central de inserção periférica" ("peripherally inserted central catheter"). Esses descritores foram combinados entre si pelos operadores booleanos "AND" e/ou "OR” e NOT. Para a seleção dos artigos, realizaram-se buscas nas bases de dados: Scientific Eletronic Library Online (SciELO), Literatura Latino-Americana em Ciências da Saúde (LILACS), PubMed.

Para facilitar a visualização do método de busca dos estudos, elaborou-se um fluxograma do tipo Prisma (Figura 1).

Figura 1: Fluxograma do método de busca dos estudos.

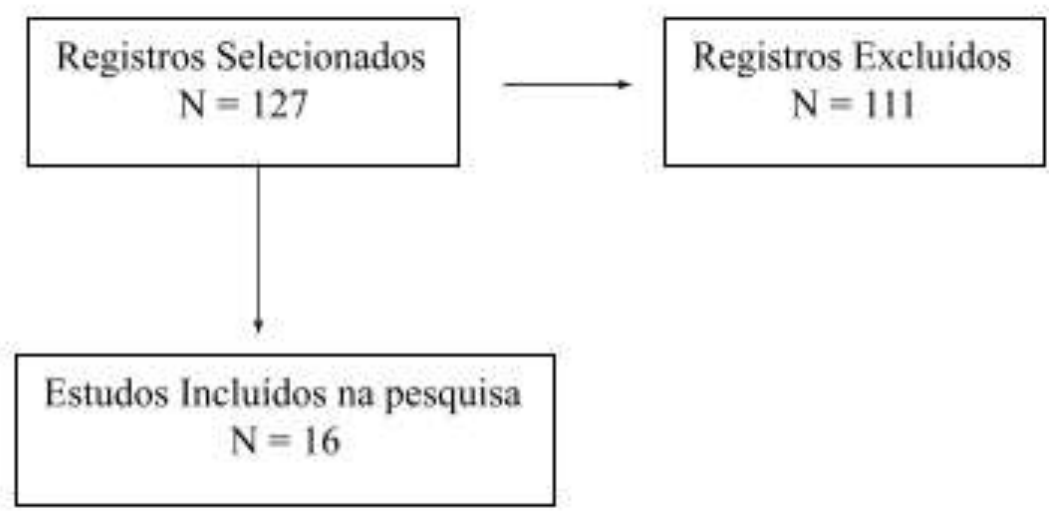

Fonte: Própria autora (2021).

Os estudos foram selecionados primeiramente pelo título e pelo resumo, gerando um total de 127 estudos. Após uma leitura na íntegra, refinou-se a amostragem, excluindo 111 por não corresponderem ao foco temático da questão norteadora. Assim, dos 127 artigos iniciais 16 foram utilizados nesta pesquisa.

Após a conclusão de todas as etapas da RI, a síntese do conhecimento sobre a temática investigada forneceu subsídios 
para a adequada análise das literaturas atuais com relação ao cateter central de inserção periférica e seu uso em pacientes neonatos.

\section{Resultados e Discussão}

Após a pesquisa, os trabalhos revisados foram organizados em forma de Quadro, separados por nomenclatura usada na pesquisa, título, objetivo, método, nível de evidência e grau de recomendação. Para nivelar a evidência e, graduar a recomendação dos artigos revisados foi utilizado o método de Nível de Evidência Científica por Tipo de Estudo - Oxford Centre for Evidencebased Medicine (Centre for Evidence-Based Medicine, 2014).

Quadro 1: Resultados da Revisão Integrativa.

\begin{tabular}{|c|c|c|c|c|c|}
\hline $\mathbf{N}$ & Título & Objetivo & Método & $\begin{array}{l}\text { Nível de } \\
\text { Evidência }\end{array}$ & $\begin{array}{c}\text { Grau de } \\
\text { Recomendação }\end{array}$ \\
\hline E1 & $\begin{array}{c}\text { Fatores de risco para infecção de } \\
\text { corrente sanguínea associada ao } \\
\text { cateter central de inserção periférica } \\
\text { em neonatos }\end{array}$ & $\begin{array}{l}\text { Identificar os fatores de risco para infecção de } \\
\text { corrente sanguínea associada ao cateter central de } \\
\text { inserção periférica em neonatos. }\end{array}$ & $\begin{array}{l}\text { Revisão sistemática de } \\
\text { ensaios clínicos } \\
\text { controlados } \\
\text { randomizados. }\end{array}$ & $1 \mathrm{~A}$ & A \\
\hline E2 & \begin{tabular}{|c|} 
Randomized Controlled Trial \\
Comparing Different Single Doses of \\
Intravenous Paracetamol for \\
Placement of Peripherally Inserted \\
Central Catheters in Preterm Infants
\end{tabular} & $\begin{array}{l}\text { To determine the analgesic effects of different } \\
\text { single intravenous paracetamol doses on pain from } \\
\text { peripherally inserted central catheter (PICC) } \\
\text { placement in preterm infants. }\end{array}$ & $\begin{array}{c}\text { Ensaio clínico controlado } \\
\text { randomizado com } \\
\text { intervalo de confiança } \\
\text { estreito. }\end{array}$ & 1B & A \\
\hline E3 & $\begin{array}{l}\text { Cost-effectiveness of strategies } \\
\text { preventing late-onset infection in } \\
\text { preterm infants. }\end{array}$ & $\begin{array}{l}\text { Developing a model to analyses the cost } \\
\text { effectiveness of interventions preventing late-onset } \\
\text { infection (LOI) in preterm infants and applying it to } \\
\text { the evaluation of antimicrobial impregnated } \\
\text { peripherally inserted central catheters (AM-PICCs) } \\
\text { compared with standard PICCs (S-PICCs). }\end{array}$ & \begin{tabular}{|} 
Observação de resultados \\
terapêuticos (outcomes \\
research). \\
Estudo Ecológico.
\end{tabular} & $2 \mathrm{C}$ & B \\
\hline E4 & $\begin{array}{c}\text { Costo de recolocación de catéter } \\
\text { central inserción periférica en } \\
\text { neonatos de un hospital de tercer } \\
\text { nivel. }\end{array}$ & $\begin{array}{l}\text { Determinar los costos por recolocación de catéter } \\
\text { central de inserción periférica (PICC) por } \\
\text { complicaciones mecánicas em neonatos de un } \\
\text { hospital de tercer nivel. }\end{array}$ & \begin{tabular}{|c|} 
Observação de resultados \\
terapêuticos (outcomes \\
research). \\
Estudo Ecológico.
\end{tabular} & $2 \mathrm{C}$ & B \\
\hline E5 & $\begin{array}{l}\text { Manejo da dor na utilização do cateter } \\
\text { central de inserção periférica em } \\
\text { neonatos. }\end{array}$ & $\begin{array}{c}\text { Descrever as práticas da equipe de enfermagem no } \\
\text { manejo da dor em recém-nascidos submetidos à } \\
\text { inserção do cateter central de inserção periférica } \\
\text { (PICC) em Unidade de Terapia Intensiva Neonatal } \\
\text { (UTIN). } \\
\end{array}$ & $\begin{array}{c}\text { Observação de resultados } \\
\text { terapêuticos (outcomes } \\
\text { research). } \\
\text { Estudo Ecológico. }\end{array}$ & $2 \mathrm{C}$ & B \\
\hline E6 & $\begin{array}{l}\text { Fibrin sheath of a peripherally } \\
\text { inserted central catheter undepicted } \\
\text { with grayscale (real-time B-mode) } \\
\text { ultrasonography: A case report. }\end{array}$ & $\begin{array}{l}\text { Demonstrate the use of color Doppler and } \\
\text { compression technique to detect fibrin sheath. }\end{array}$ & $\begin{array}{c}\text { Relato de Casos } \\
\text { (incluindo coorte ou } \\
\text { caso-controle de menor } \\
\text { qualidade). }\end{array}$ & 4 & $\mathrm{C}$ \\
\hline E7 & $\begin{array}{l}\text { Custo direto da passagem de cateter } \\
\text { central de inserção periférica por } \\
\text { enfermeiros. }\end{array}$ & $\begin{array}{l}\text { Mensurar o custo direto médio da passagem de } \\
\text { cateter central de inserção periférica, por } \\
\text { enfermeiros, em uma unidade de terapia intensiva } \\
\text { pediátrica e neonatal. }\end{array}$ & $\begin{array}{c}\text { Observação de resultados } \\
\text { terapêuticos (outcomes } \\
\text { research). } \\
\text { Estudo Ecológico. }\end{array}$ & $2 \mathrm{C}$ & B \\
\hline E8 & $\begin{array}{c}\text { Tamponamento cardíaco por cateter } \\
\text { central de inserção periférica em } \\
\text { prematuros: papel da ultrassonografia } \\
\text { à beira do leito e abordagem } \\
\text { terapêutica. }\end{array}$ & $\begin{array}{c}\text { Determinar a incidência de derrame pericárdico } \\
\text { com tamponamento cardíaco em recém-natos } \\
\text { prematuros em uma unidade de terapia intensiva } \\
\text { pediátrica, com ênfase na relação entre o derrame } \\
\text { pericárdico e a inserção de cateter central de } \\
\text { inserção periférica, e avaliar o papel da } \\
\text { ultrassonografia à beira do leito na abordagem } \\
\text { desses casos. }\end{array}$ & $\begin{array}{c}\text { Observação de resultados } \\
\text { terapêuticos (outcomes } \\
\text { research). } \\
\text { Estudo Ecológico. }\end{array}$ & $2 \mathrm{C}$ & B \\
\hline E9 & $\begin{array}{c}\text { Avaliação da dor de recém-nascidos } \\
\text { durante procedimentos invasivos em } \\
\text { terapia intensiva. }\end{array}$ & $\begin{array}{l}\text { Avaliar a dor de recém-nascidos internados em } \\
\text { unidade de terapia intensiva neonatal durante a } \\
\text { realização de procedimentos invasivos. }\end{array}$ & \begin{tabular}{|c|}
$\begin{array}{c}\text { Observação de resultados } \\
\text { terapêuticos (outcomes } \\
\text { research). } \\
\text { Estudo Ecológico. }\end{array}$ \\
\end{tabular} & $2 \mathrm{C}$ & B \\
\hline E10 & $\begin{array}{l}\text { The Extended Dwell Peripheral } \\
\text { Intravenous Catheter Is an Alternative } \\
\text { Method of NICU Intravenous Access }\end{array}$ & $\begin{array}{l}\text { Explore indwell time, success rate, catheter- } \\
\text { associated complications, and cost among EPIV } \\
\text { catheters, PICCs, and PIV catheters in neonates. }\end{array}$ & \begin{tabular}{|c|} 
Estudo de Coorte \\
(incluindo Ensaio \\
Clínico Randomizado de \\
menor qualidade).
\end{tabular} & $2 \mathrm{~B}$ & B \\
\hline
\end{tabular}




\begin{tabular}{|c|c|c|c|c|c|}
\hline E11 & \begin{tabular}{|c|} 
Variation in infection prevention \\
practices for peripherally inserted \\
central venous catheters: A survey of \\
neonatal units in England and Wales.
\end{tabular} & $\begin{array}{l}\text { To evaluate evidence supporting infection } \\
\text { prevention practices for CVCs recommended in } \\
\text { national guidelines and to compare with reported } \\
\text { practices for peripherally inserted central catheters } \\
\text { (PICC), a type of CVC widely used in NNUs. }\end{array}$ & $\begin{array}{l}\text { Revisão sistemática de } \\
\text { ensaios clínicos } \\
\text { controlados } \\
\text { randomizados. }\end{array}$ & $1 \mathrm{~A}$ & A \\
\hline E12 & $\begin{array}{l}\text { Variáveis associadas a eventos } \\
\text { adversos em neonatos com cateter } \\
\text { central de inserção periférica. }\end{array}$ & $\begin{array}{c}\text { Identificar os fatores associados à ocorrência de } \\
\text { eventos adversos por cateter central de inserção } \\
\text { periférica em recém-nascidos. }\end{array}$ & $\begin{array}{c}\text { Observação de resultados } \\
\text { terapêuticos (outcomes } \\
\text { research). } \\
\text { Estudo Ecológico. }\end{array}$ & $2 \mathrm{C}$ & B \\
\hline E13 & $\begin{array}{l}\text { Caracterização física de Cateteres } \\
\text { Centrais de Inserção Periférica }\end{array}$ & $\begin{array}{l}\text { Avaliar as características físicas de CCIP, por meio } \\
\text { da aplicação das normas ABNT NBR ISO 10555-1: } \\
2003 \text { e ABNT NBR ISO 10555-3: 2003, e } \\
\text { comparar os resultados produzidos na análise de } \\
\text { CCIP no início de seu processo de produção } \\
\text { (somente cateteres extrusados) com os obtidos na } \\
\text { análise do produto acabado. }\end{array}$ & $\begin{array}{l}\text { Resultados terapêuticos } \\
\text { do tipo "tudo ou nada". }\end{array}$ & $1 \mathrm{C}$ & A \\
\hline E14 & \begin{tabular}{|} 
Improved visualization of \\
peripherally inserted central catheters \\
on chest radiographs of neonates \\
using fractional multiscale image \\
processing.
\end{tabular} & $\begin{array}{c}\text { The aim of this study is to evaluate the ability of } \\
\text { Fractional Multiscale image Processing (FMP) to } \\
\text { detect PICC tips on the digital chest radiographs of } \\
\text { neonates. }\end{array}$ & $\begin{array}{l}\text { Opinião de especialistas } \\
\text { desprovida de avaliação } \\
\text { crítica. }\end{array}$ & 5 & D \\
\hline E15 & $\begin{array}{l}\text { Use of real-time ultrasound for } \\
\text { locating tip position in neonates } \\
\text { undergoing peripherally inserted } \\
\text { central catheter insertion: A pilot } \\
\text { study. }\end{array}$ & $\begin{array}{l}\text { This study was done to compare the effectiveness } \\
\text { of real-time ultrasound (RTUS) with X-ray in } \\
\text { identifying the peripherally inserted central catheter } \\
\text { (PICC) line tip. }\end{array}$ & $\begin{array}{c}\text { Ensaio clínico controlado } \\
\text { randomizado com } \\
\text { intervalo de confiança } \\
\text { estreito. }\end{array}$ & $1 \mathrm{~B}$ & A \\
\hline E16 & $\begin{array}{c}\text { Selection of PICC catheter location in } \\
\text { neonates via evidence-based ACE } \\
\text { Star model. }\end{array}$ & $\begin{array}{c}\text { To explore the most suitable insertion site for } \\
\text { neonatal placement of peripherally inserted central } \\
\text { venous catheter (PICC) according to the evidence- } \\
\text { based ACE Star model. }\end{array}$ & $\begin{array}{c}\text { Observação de resultados } \\
\text { terapêticos (outcomes } \\
\text { research). }\end{array}$ & $2 \mathrm{C}$ & B \\
\hline
\end{tabular}

Fonte: Produção própria (2021).

Dos estudos analisados, 16 abordaram a temática da pesquisa. Desses, 3 (18,75\%) foram publicados em 2016, 3 (18,75\%) em 2017, 5 (31,25\%) em 2018, 3 (18,75\%) em 2019 e 2 (12,50\%) em 2020. Verificou-se que, de forma unânime, os artigos selecionados tratam de diminutas temáticas em relação ao produto da utilização do cateter central de inserção periférica em neonatologia.

Após análise minuciosa dos artigos estudados, foram segregados em categorias estabelecidas levando em consideração seus objetivos, sendo que as categorias geradas foram: Complicações causadas pelo uso do cateter central de inserção periférica (CCIP) e custo com o uso do cateter.

\section{Complicações causadas pelo uso do cateter central de inserção periférica (CCIP)}

As complicações causadas pelo uso do CCIP podem variar de mínimas complexas que podem conferir risco de morte ao paciente. Essas iatrogenias foram evidenciadas em 12 artigos dispostos a seguir no Quadro 2, que apresenta as principais complicações descritas pelos autores estudados.

Quadro 2. Principais iatrogenias que podem ser causadas pela utilização de CCIP.

\begin{tabular}{|l|c|c|}
\hline Evento Adverso & N de artigos (\%) & Artigos \\
\hline Mal posicionamento do cateter & $5(41,66 \%)$ & E6, E8, E14, E15 e E16 \\
\hline Dor & $3(33,33 \%)$ & E2, E5, E9 e E10 \\
\hline Infecção de corrente sanguínea & $2(16,66 \%)$ & E1 e E11 \\
\hline Outros & $1(8,33 \%)$ & E12 \\
\hline
\end{tabular}

Fonte: Própria autora (2021). 
Os pacientes que necessitam do uso de um cateter central, são clientes que normalmente estão fazendo uso de antibioticoterapia, uso de medicações vesicantes ou até mesmo não têm mais condições de acesso periférico (Prado et al, 2020). Neonatos em unidades de terapia intensiva são candidatos a esse procedimento por terem uma rede venosa mais débil e que necessita de maior cuidado, conforme exemplificado no E10 (Chenoweth, Guo, \& Chan, 2018).

Prado et al. (2020), E12, ainda discorrem que as principais variáveis associadas ao desenvolvimento de intercorrências foram sexo do bebê, havendo maior prevalência no sexo masculino, peso, com mais de 48 horas de vida, número de tentativas de inserção do cateter, superior a três, presença de intercorrências durante a inserção do PICC, número de trocas do curativo acima de duas, dificuldade de progressão do cateter e localização não central do dispositivo.

A escolha do local para inserção desse cateter, pode também influenciar entre outras possíveis complicações relacionados no E16 (Chen, Zhou, Tan, \& Tao, 2020). Outro evento adverso que podemos encontrar no procedimento da passagem de cateter central de inserção periférica é a dor. Como qualquer procedimento invasivo a dor pode e, estará presente, é conhecido pela equipe que o procedimento em questão, gera dor nos pacientes como é descrito no E5 (Kegler, Paula, Neves, \& Jantsch, 2016).

A equipe de enfermagem reconhece que a inserção do PICC é um procedimento doloroso, o que justifica a utilização de práticas analgésicas. Ainda, os participantes citaram como justificativas para adoção de analgesia, a necessidade de manter o RN calmo ao longo do procedimento, a atenuação da dor e a redução do estresse profissional (Kegler et al., 2016).

No E9 a avaliação da dor foi realizada através de escalas específicas, sendo que como resultado, obteve-se que durante a passagem do CCIP a dor variou de moderada a intensa nos neonatos (Cruz, Gomes, Kirchne, \& Stumm, 2016).

Mesmo que não seja possível anular procedimentos invasivos dolorosos, os mesmos podem ser controlados por métodos farmacológicos, ou não farmacológicos. O E2 traz duas opções farmacológicas para antes de procedimentos dolorosos agudos em bebês prematuros (Roofthood et al, 2017). No estudo em questão foi utilizado o paracetamol endovenoso, não disponível ainda no Brasil, para analgesia do paciente, sendo o fármaco com eficácia aquém à sacarose. Para alívio da dor as doses deveriam ser superiores a $10 \mathrm{mg} / \mathrm{kg}$, não sendo a droga escolhida para este ou outros procedimentos no qual há lise de tegumentos em prematuros.

Além da dor causada pela inserção do cateter, outra iatrogenia que pode ocorrer é o tamponamento cardíaco. Mesmo que um evento raro o E8, afirma que a simples utilização de ultrassonografia beira leito pode diagnosticar o derrame pericárdico (Barreiros et al., 2018). O E14 indica a utilização de radiografias sequenciais à beira leito para verificação da progressão do cateter (Hammon et al., 2019), todavia, os E6 e E15 reafirmam que a utilização de ultrassonografia em tempo real é uma ferramenta na identificação de posições de linha CCIP e que, se usada de forma adequada, evita exposições do neonato a raiosX (Telang, Sharma, Pratap, Kandraju, \& Murki, 2017; Mogi et al., 2018).

Como intervenção, Barreiros et al. (2018) sugerem a realização de uma pericardiocentese, estabilizando hemodinamicamente o paciente de forma instantânea. Porém, no caso de suspeita de perfuração cardíaca deve-se realizar uma pericardiocentese e, retirada ou tração do cateter central para melhor resolução do quadro.

As infecções de corrente sanguínea associadas ao cateter central de inserção periférica em neonatos, são uma das maiores preocupações dos profissionais de saúde (Costa, Paiva, Kimura, \& Castro, 2016). Sendo que, as variáveis que mais concomitam em possíveis infecções de corrente sanguínea são a menor idade gestacional corrigida do neonato, os diagnósticos clínicos de transtorno transitório do metabolismo, apneia, diagnósticos graves que necessitam de maiores trocas de terapias infusionais e, o uso do cateter de duas vias como aponta o E1.

O E11 aponta a utilização de um conjunto de cuidados com cateteres centrais entre eles o uso de curativo impregnado de clorexidina, remoção de rotina ou substituição de CCIP. Consideram esta última recomendação mais eficaz que o uso de clorexidina alcoólica $2 \%$ para preparação da pele e desinfecção portuária, pois a clorexidina pode causar queimaduras na pele 
dos neonatos (Fraser, Harron, Dalton, Gilbert, \& Oddie, 2018).

\section{Custo com o uso do cateter}

O custo com a utilização do cateter central de inserção periférica assim como, outros procedimentos nos quais são utilizados materiais especiais, tem um custo expressivo em decorrência do valor despendido para a compra das órteses, próteses e materiais especiais (OPME). O E7 realizou um estudo em unidades de terapia intensiva pediátrica e neonatal de um hospital público de ensino e pesquisa no qual obteve o valor do custo médio direto do procedimento passagem de CCIP por enfermeiros correspondeu a US\$326.95, R \$ $1.787,47^{1}$ aproximadamente, valor esse, representado pelo honorário da equipe de enfermagem, custo com o material, soluções e medicamentos (Pires \& Lima, 2019).

Além do custo direto com a inserção do cateter, existem outros fatores que podem encarecer o procedimento, sendo um deles as complicações já citadas anteriormente. No E3 as infecções tardias que podem acarretar o comprometimento neuropsicomotor, custam cerca de $£ 19$ 057, R \$ 142.543,73² aproximadamente (Grosso et al., 2019). Haja visto que essa complicação concomita com maiores períodos de internação em unidades de terapia intensiva.

O E4, que foi realizado em Tabesco, no México, registrou 43 incidentes com cateteres centrais de inserção periférica, sendo 22 casos de ruptura, 20 casos por obstrução e 1 caso por retirada acidental. Estes incidentes resultaram em recolocações dos cateteres custando um valor unitário para o procedimento de U\$3387.80, R\$ 104.186,52 ${ }^{2}$ aproximadamente (Aguayo, Hernandes, \& Padron, 2019).

Aguayo, Hernandes e Padron (2019) observam que existe uma cadeia de procedimentos e intervenções que geram altos custos quando há um evento adverso durante o uso do cateter central de inserção periférica, sendo o principal, a extensão de dias de internação. Tendo em vista que os hospitais não alocam parte do orçamento para cobrir valores causados por essas iatrogenias, existem prejuízos aos orçamentos das instituições de saúde.

O E10, Chenoweth, Guo \& Chan (2018) trazem que o custo do trabalho é calculado com base no salário médio de enfermagem de US\$30 por hora, $\mathrm{R} \$ 161,08^{2}$ aproximadamente. Um mínimo de dois profissionais graduados em enfermagem é tipicamente necessário para inserir um CCIP exigindo então de 60 min em média para a inserção do cateter, duas radiografias para confirmação do posicionamento do mesmo, gerando um custo semanal de US\$ 517, R $\$ 2.826,49^{2}$ aproximadamente, valor extremamente elevado quando comparado a um cateter periférico comumente utilizado em internações.

\section{Conclusão}

Concluiu-se pelos estudos avaliados que a utilização do cateter central de inserção periférica em neonatologia é de extrema importância em unidades de terapia intensiva. Entretanto, mesmo que de suma importância terapêutica, a utilização desse material especial pode acarretar diversos eventos adversos quando realizado de forma errônea. O mal posicionamento foi a complicação mais relatada nos artigos estudados, por dor e infecções de corrente sanguínea.

O elevado custo do procedimento é um assunto muito salientado nos artigos estudados, todavia não foram encontrados artigos que calculassem esse custo no Brasil, todos utilizaram valores internacionais.

Diante da escassez de literatura sobre sua indicação, contraindicação e melhor localização para sua instalação. E visto a sua portanto a sua importância no meio intensivista, sugere-se pesquisa e estudos mais amplos a respeito da utilização de CCIP em neonatologia.

\footnotetext{
1 Valores convertidos tendo como base de referência valor do dólar $\mathrm{R} \$ 5,37$.

2 Valor convertido tendo como base de referência valor da libra esterlina $\mathrm{R} \$ 7,35$.
} 


\section{Referências}

Aguayo, K.I.L., Hernandez, C.M.M., \& Padron, H.S. (2019). Costo de recolocación de catéter central inserción periférica en neonatos de un hospital de tercer nivel. Horizontal Sanitário, 18(1), 49-55.

Barreiros, L.L., Andrade, F.M., Torres, R.A., Magalhães, L.V.B., Farnetano, B.S., \& Fiorelli, R.K.A. (2018). Tamponamento cardíaco por cateter central de inserção periférica em prematuros: papel da ultrassonografia à beira do leito e abordagem terapêutica. Revista do Colégio Brasileiro de Cirurgia, 45(3), e1818.

Chen, X., Zhou, L.,Tan, Y., \& Tao, Z. (2020). Selection of PICC catheter location in neonates via evidence-based ACE Star model. Journal of Central South University: Medical Sciences, 9(2845), 1082-88.

Chenoweth, K.B., Guo JW, \& Chan, B. (2018). The Extended Dwell Peripheral Intravenous Catheter Is an Alternative Method of NICU Intravenous Access. Advances in Neonatal Care, 18(4), 295-301.

Comissão Nacional de Incorporação de Tecnologias do SUS. (2014). Nível de Evidência Científica por Tipo de Estudo - Oxford Centre for Evidence-based Medicine. São Paulo: CNITEC.

Conselho Federal de Enfermagem. (2017). Parecer $n^{\circ}$ 243. Atualiza a normatização do procedimento de inserção, fixação, manutenção e retirada de cateter periférico central (PICC) por enfermeiro. Brasília: Cofen.

Costa, P., Paiva, E.D., Kimura, A.F., \& Castro, T.E. (2016). Fatores de risco para infecção de corrente sanguínea associada ao cateter central de inserção periférica em neonatos. Acta Paulista de Enfermagem, 29(2), 161-8.

Cruz, C.T., Gomes, J.S., Kirchner, R.M., \& Stumm, E.M.F. (2016). Avaliação da dor de recém-nascidos durante procedimentos invasivos em terapia intensiva. Revista Dor, 17(3), 197-200.

Fraser, C., Harron, K., Dalton, L., Gilbert, R., \& Oddie, S.J. (2018). Variation in infection prevention practices for peripherally inserted central venous catheters: a survey of neonatal units in England and wales. Plos One, 13(11), 1-13.

Fricke, B.L., Racadio, J.M., Duckworth, T., Donnelly, L.F., Tamer, R.M., \& Johnson, N.D. (2005). Placement of Peripherally Inserted Central Catheters without Fluoroscopy in Children: initial catheter tip position. Radiology, 234(3), 887-92.

Grosso, A., Faria, R.I.N., Bojke, L., Donohue, C., Fraser, C.I., Harron, K., et al. (2019). Cost-effectiveness of strategies preventing late-onset infection in preterm infants. Archives of Disease in Childhood, 105(5), 452-7.

Hammon, R.A., Seuss, H., Hammon, M., Grillhös, C., Heiss, R., Zeilinger, M., et al. (2019). Improved visualization of peripherally inserted central catheters on chest radiographs of neonates using fractional multiscale image processing. BMC Medical Imaging, 19(1), 1-7.

Kegler, J.J., Paula, C.C., Neves,E.T., \& Jantsch, L.B. (2016). Manejo da dor na utilização do cateter central de inserção periférica em neonatos. Escola Anna Nery, 20(4), e20160099.

Minayo, M.C.S. (2013). Pesquisa social: Teoria, método e criatividade. 33. ed. Petrópolis: Vozes.

Mitelmão, F.C.R., Mangini Filho, S., Chaud, M.V., Vila, M.M.D.C., Balcão, V.M.C.F., \& Oliveira Junior, J.M. (2020). Caracterização física de Cateteres Centrais de Inserção Periférica (CCIP). Matéria, 25(1), e-12545.

Mogi, N., Nakagawa, M., Matsumae, H., Hattori, A., Shimohira, M., \& Shibamoto, Y. (2018). Fibrin sheath of a peripherally inserted central catheter undepicted with gray-scale (real-time B-mode) ultrasonography: a case report. Radiology Case Reports, 13(3), 537-41.

Philpot, P., \& Griffiths, V. (2003). The peripherally inserted central catheter. Nursing Standard, 17(4), 39-46.

Pires, A.B.M., \& Lima, A.F.C. (2019). Custo direto da passagem de cateter central de inserção periférica por enfermeiros. Revista Brasileira de Enfermagem 72(1), 88-94.

Prado, N.C.C., Santos, R.S.C., Almino, R.H.S.C., Lima, D.M., Oliveira, S.S., \& Silva, R.A.R. (2020). Variáveis associadas a eventos adversos em neonatos com cateter central de inserção periférica. Enfermería Global, 19(3), 36-67.

Roofthooft, D.W.E., Simons, S.H.P., Lingen, R.A., Tibboel, D., Anker, J.N.A., Reiss, I.K.H., \& Dijk, M. (2017). Randomized Controlled Trial Comparing Different Single Doses of Intravenous Paracetamol for Placement of Peripherally Inserted Central Catheters in Preterm Infants. Neonatology, 112(2), 150-8.

Souza, M.T., Silva, M.D., \& Carvalho, R. (2010). Revisão integrativa: o que é e como fazer. Einstein, 8(1), 102-6.

Telang, N., Sharma, D., Pratap, O.T., Kandraju, H., \& Murki, S. (2017). Use of real-time ultrasound for locating tip position in neonates undergoing peripherally inserted central catheter insertion: a pilot study. Indian Journal of Medical Research, 3(145), 373-6.

Toma, E., \& Tsunechiro, M.A. (2004). Avaliação do uso do Cateter Central de Inserção Periférica (PICC) em recém-nascidos. São Paulo: Universidade de São Paulo. 